\title{
Near-field cosmology with Local Group dwarf spheroidals
}

\author{
Eva K. Grebel \\ Astronomical Institute, University of Basel, CH-4102 Binningen, Switzerland \\ email: grebel@astro.unibas.ch
}

\begin{abstract}
The Local Group offers an excellent laboratory for near-field cosmology by permitting us to use the resolved stellar content of its constituent galaxies as probes of galaxy formation and evolution, which in turn is an important means for testing cosmological models of hierarchical structure formation. In this review, we discuss the the least massive, yet most numerous type of galaxy in the Local Group, the dwarf spheroidal galaxies, and compare their properties to cosmological predictions. In particular, we point out problems found with a simple building block scenario and with effects expected from reionization. We show that the star formation histories of $\mathrm{dSphs}$ are inconsistent with the predicted cessation of star formation after reionization; instead, extended star formation episodes are observed. The Galactic dSphs contain in part prominent intermediate-age populations, whereas the Galactic halo does not. Conversely, the M31 dSphs are almost entirely old, while the M31 halo contains a substantial intermediate-age population. These differences in the population structure as well as the differences in the modes of star formation inferred from $[\alpha / \mathrm{Fe}]$ ratios make $\mathrm{dSphs}$ unlikely major contributors to the build-up of the Galactic and M31 halo unless most of the accretion occurred at early epochs. On the other hand, there is clear evidence for ongoing harassment and accretion of a number of dSphs.
\end{abstract}

Keywords. galaxies: Local Group, galaxies: dwarf, galaxies: evolution, galaxies: structure, galaxies: interactions, cosmology: observations, Galaxy: formation, Galaxy: evolution, stars: latetype, stars: abundances.

\section{Introduction}

The Local Group is an excellent laboratory for studies of galaxy evolution at the highest possible resolution in that it provides us with a wide range of different galaxy types and a variety of environments. Yet the Local Group is a poor group, contains relatively few galaxies, and lacks very massive galaxies, such as large ellipticals. Similar to many other nearby groups, the mass and the luminosity of the Local Group are dominated by two large spirals, the Milky Way and M31. Most of the other Local Group members are dwarf galaxies, and the majority of them are found in close proximity to the two large spirals.

Dwarf galaxies are often considered building blocks of more massive galaxies in models of hierarchical structure formation. Dwarf galaxies come in many different flavors and cover a range of masses, luminosities, morphologies, gas content, star formation histories, etc. The distinction between dwarf galaxies and larger galaxies is somewhat fuzzy. The difference is primarily a luminosity difference - it is customary to call galaxies with absolute magnitudes of $M_{V}>-18$ dwarf galaxies. Gas-rich dwarfs include dwarf spirals, dwarf irregulars (dIrrs) and blue compact dwarf galaxies, which usually show differing levels of ongoing star formation. Gas-poor dwarfs are primarily dwarf ellipticals (dEs). These can be further subdivided into subtypes such as the more massive, strongly centrally concentrated dwarf ellipticals with higher surface brightness, and the less massive, faint, fairly diffuse dwarf spheroidals (dSphs) (see also Gallagher \& Wyse 1994; Grebel, Gallagher, \& Harbeck 2003). 
What makes the Local Group special (apart from its being our home) is that here we have the possibility to actually resolve its constituent galaxies into individual stars and to study the properties of these stars. We can use these stars as probes of the past - they permit us to uncover the evolutionary histories of their host galaxies. Moreover, they permit us to study these evolutionary histories at a level of detail and accuracy that is unmatched by any more distant galaxy, where only the integrated light can be studied. The Local Group is the only place where we can analyze even ancient stars and uncover the early formation history of individual galaxies beyond our own, particularly of the dwarf companions of the Milky Way. To summarize, the Local Group is ideally suited for studies of "near-field cosmology", i.e., for studies of galaxy evolution over cosmological epochs based on their resolved stellar fossil record, and for tests of the corresponding cosmological models.

\section{Local Group Dwarf Spheroidals}

The galaxy census of the Local Group remains uncertain. Within the Local Group's volume as defined by its zero velocity surface of $\sim 1 \mathrm{Mpc}$ (Karachentsev et al. 2002), we currently know of some 38 probable member galaxies. Some were only recently discovered, and additional faint candidates continue to be found (e.g., Zucker et al. 2004a,b). All of the newly discovered dwarfs are dSphs, the least massive, least luminous galaxies known, and thus contribute to the faint end of the galaxy luminosity function. For reviews on Local Group dwarfs, see, e.g., Grebel (1997, 1999, 2000, 2001, 2005), Mateo (1998), and van den Bergh (1999, 2000).

DSphs are usually the most numerous type of galaxy in galaxy groups and are characterized by $\mathrm{M}_{V}<-14 \mathrm{mag}, \mu_{V}<22 \mathrm{mag} \operatorname{arcsec}^{-2}, M_{\mathrm{HI}}<10^{5} \mathrm{M}_{\odot}$, and $M_{\text {tot }} \sim 10^{7} \mathrm{M}_{\odot}$. Often their stellar populations are purely old, but mixtures of old and intermediate-age populations are found as well. In dSphs where several populations can be distinguished the younger and/or more metal-rich populations are more centrally concentrated, indicating extended star formation episodes in the centers of the shallow potential wells of their parent galaxies (Harbeck et al. 2001). The gas deficiency of dSphs remains an unsolved puzzle - dSphs typically contain even less gas than expected from red giant mass loss over time scales of several Gyr. The metallicity-luminosity relations of dSphs and dIrrs show the usual trend of increasing metallicity with increasing galaxy luminosity, but the relations are offset from each other: DSphs have higher mean stellar metallicities at a given optical luminosity, which may indicate more rapid star formation and enrichment at early times as compared to dIrrs (Grebel et al. 2003).

DSphs do not seem to be supported by rotation and appear to contain large amounts of dark matter. The latter is inferred from the high velocity dispersion and the resulting high mass-to-light ratios derived under the assumption of virial equilibrium. Indirectly, a high dark matter content is also supported by the morphology of some nearby dSphs (Odenkirchen et al. 2001) and by the observed lack of a significant depth extent (Klessen, Grebel, \& Harbeck 2003). The radial velocity dispersion profiles of dSphs fall off large radii (Wilkinson et al. 2004), possibly indicating the presence of a kinematically cold stellar population at the outermost radii.

If dwarf galaxies in general and dSphs in particular are indeed building blocks of larger galaxies, then today's dwarf population may be considered to be the surviving population of satellites that has not yet been accreted. The most numerous type of dwarfs in galaxy groups, the dSphs, may then be the most pristine members of the original building block population. Studying dSphs may teach us about the properties of objects that presumably were once accreted in large numbers to form galaxies like the Milky Way. Alternatively, it 
is conceivable that dSphs are in fact not fossil building blocks, but stripped remnants of disrupted and originally much more massive galaxies that have since merged. To find out more about the nature of dSphs and their cosmological significance we need to understand their past and present-day properties.

\section{Dwarf Spheroidals: The Earliest Measurable Epoch of Star Formation and Its Cosmological Implications}

Cold dark matter models predict that low-mass systems were the first sites of star formation, possibly as early as at a redshift of 30 (e.g., Barkana \& Loeb 2001). Since larger systems form through hierarchical merging of smaller systems, they should contain surviving populations of these early epochs of star formation. Furthermore, several models predict that small galaxies should have formed most of their stars prior to reionization, while reionization would have suppressed further star formation activity. In fact, galaxies less massive than $10^{9} \mathrm{M}_{\odot}$ should have lost their star-forming material during reionization (e.g., Susa \& Umemura 2004). Hence one would expect that low-mass galaxies contain ancient populations, while star formation should have ceased after reionization.

These are predictions that can be tested in the dwarfs in our immediate surroundings. The least massive dwarfs, the dSphs, should have been most severely affected. Deep color-magnitude diagram data of these dwarfs that reach below the oldest main-sequence turn-offs permit us to carry out relative age dating of their old populations with internal accuracies of fractions of $\sim 1$ Gyr, the highest accuracy currently attainable for any method for old stars. Note that this method can only be applied to populations sufficiently numerous to form detectable main-sequence turn-offs. This holds only for old Population II stars, while potential Population III stars are far too few even in our Milky Way. The differential ages of old populations in dwarf galaxies - either field populations or globular clusters - can then be compared to the ages of the oldest Galactic globular clusters of similar composition.

Importantly, this method reveals that (1) old populations are ubiquitous (but their fractions vary) and (2) the oldest ages in all of the galaxies studied so far are indistinguishable within the measurement accuracy (see Grebel 2000 and Grebel \& Gallagher 2004 for details). All nearby dwarf galaxies studied in sufficient detail were shown to contain ancient populations. Moreover, these nearby Milky Way companions and the Milky Way itself share a common epoch of star formation for their ancient Population II (within $\sim 1$ Gyr). These observations are consistent with the expectations from the building block scenario.

However, the predicted cessation of star formation after reionization, expected to have affected particularly dSphs owing to their low mass, is not observed (Grebel \& Gallagher 2004). Instead, even dSphs entirely dominated by old populations show evidence for star formation extending over many Gyr (Harbeck et al. 2001; Ikuta \& Arimoto 2002). In dSphs with a mixture of populations we usually find star formation episodes that lasted many Gyr without being interrupted by a pronounced, long hiatus to the extent that we can measure the duration of star formation (exception: Carina with its episodic star formation). This may mean that the above quoted cosmological models are incorrect and do not properly consider other effects that might prevent complete photoevaporation (e.g., Susa \& Umemura 2004). On the other hand, photoionization is one plausible way to circumvent the substructure problem (e.g., Somerville 2002). Alternatively, it is conceivable that dSphs were once considerably more massive (by roughly a factor of 100), which could also have prevented photoionization squelching. In this case the galaxies observed today as dSphs must have undergone substantial mass loss. 


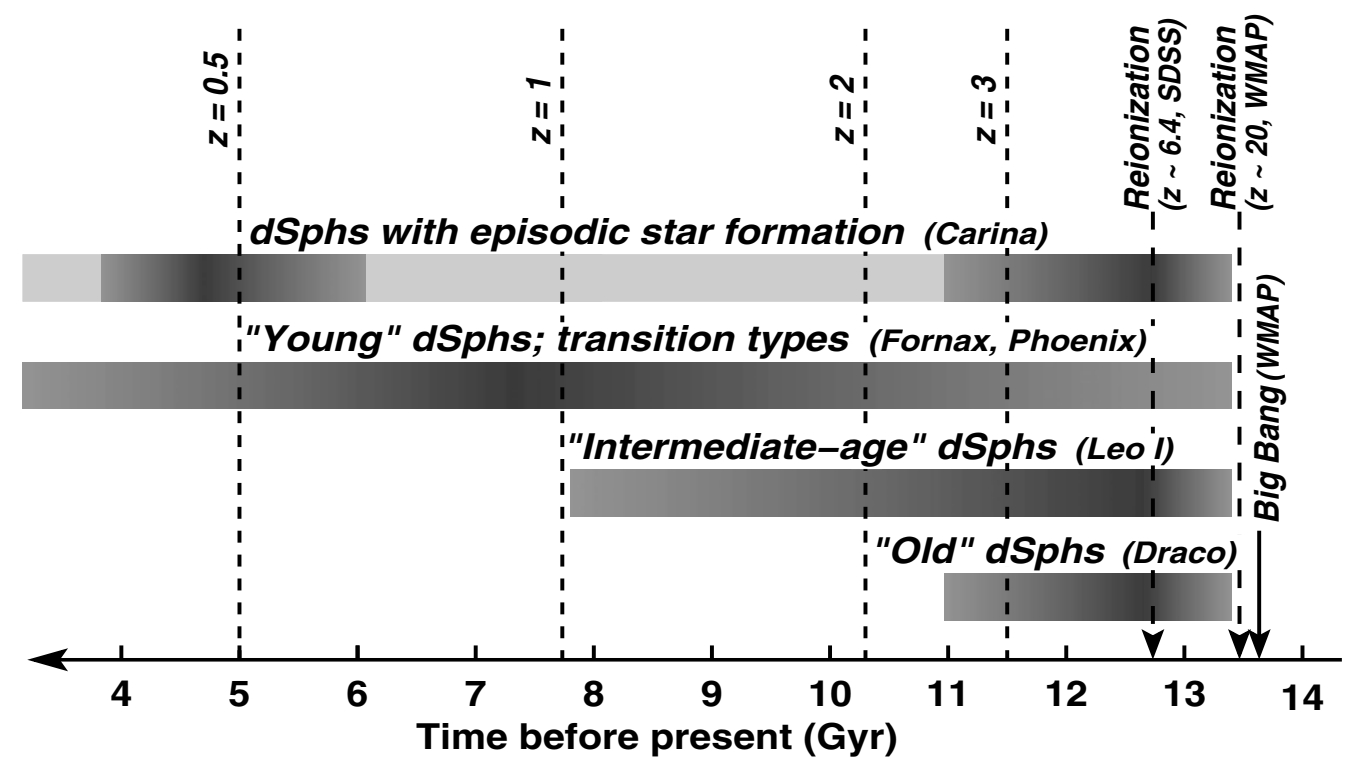

Figure 1. Sketch indicating the approximate duration of star formation episodes in dSph galaxies $\left(\sim 10^{7} \mathrm{M}_{\odot}\right)$. The adopted beginning and end of the reionization epoch are based on results from WMAP and from the Sloan Digital Sky Survey. The predicted cessation of star formation after reionization is not observed. For details, see Grebel \& Gallagher (2004).

\section{Dwarf Spheroidal Star Formation Histories and Abundances}

Thanks to deep, high-resolution photometry and synthetic color-magnitude diagram techniques, fairly detailed knowledge of the star formation histories of Local Group dSphs is now available. The resulting picture is one of high complexity: No two dSphs exhibit the same star formation history (Grebel 1997). As mentioned already, all dSphs studied in detail so far were found to contain old Population II stars. Some dSphs are dominated by ancient stars, others only have a small old population and a dominant intermediateage population, and there is one example of a dSph that experienced star formation as recently as a few hundred Myr ago (Fornax, see Grebel \& Stetson 1999). Generally, star formation has proceeded continuously in these galaxies, although the amplitude varied and eventually declined at intermediate or younger ages (e.g., Grebel et al. 2003). Only one dSph with clearly episodic star formation is known (Carina, Smecker-Hane et al. 1994 and Monelli et al. 2003). DSphs with several populations typically show population gradients in the sense that more metal-rich and/or younger populations are more centrally concentrated (Harbeck et al. 2001). Substructure of this kind is not necessarily symmetrically distributed (e.g., Stetson et al. 1998).

While the past decade was mainly one of photometrically derived star formation histories, we are now entering an era where the age-metallicity degeneracy, which is inherent to purely photometric determinations, can be broken by adding spectroscopic abundance information (e.g., Tolstoy et al. 2001; Pont et al. 2004, Cole et al. 2005, Koch et al. in these proceedings). This will ultimately permit us to derive detailed age-metallicity relations for these galaxies.

\subsection{Comparing stellar populations and star formation histories}

Comparing star formation histories of dwarf galaxies in general and dSphs in particular (e.g., Grebel 1997, 1999), one finds variations in the duration of star formation, in the 
star formation rates as a function of time, and in the enrichment. In spite of being overall metal-poor, all dSph galaxies that were studied spectroscopically so far show a spread of metallicities of typically 1 dex in $[\mathrm{Fe} / \mathrm{H}]$ or more (e.g., Shetrone et al. 2001, 2003; Bonifacio et al. 2004). There appears to be a trend of increasing intermediate-age population fractions with increasing distance from the Milky Way among the Galactic dSphs (van den Bergh 1994; Grebel 1997), which may be due to the environmental impact of the Milky Way.

If environment was indeed the governing factor determining the evolution of these lowmass galaxies, then one should expect to find a similar trend among the dSph companions of M31. However, this is not observed. Although M31's dSphs cover a comparable range of distances as their Galactic counterparts, they all appear to be dominated by old populations and lack the indicators of prominent intermediate-age populations present in the more distant Milky Way dSphs (Harbeck et al. 2001, 2004, 2005).

Considering what we can infer from present-day dSphs about their star formation histories, how do they fit in as potential building blocks? With respect to stellar populations, dSphs dominated by old populations are compatible with the stellar content of the Galactic halo. DSphs with substantial intermediate-age populations seem less likely to have made a major contribution to the build-up of the halo of our Milky Way (Unavane et al. 1996). On the other hand, this problem would be diminished if most of the minor merger events took place at very early epochs. Comparing the old, metal-poor stellar populations in M31's dSphs to M31's halo indicates that the dSphs cannot have been primary building blocks of M31's halo since it was found to contain a substantial contribution from intermediate-age, comparatively metal-rich populations (Brown et al. 2003). An old, metal-poor halo population, however, has been detected as well (Brown et al. 2004), and again the population differences would be less severe if most of the $\mathrm{dSph}$ accretion had taken place at very early epochs, whereas the remainder of the younger halo of M31 would have been formed through the later accretion of more massive and more evolved galaxies. - These statements assume that dSphs have not changed appreciably over time (e.g., did not lose substantial amounts of mass) and that their observed stellar content permits one to arrive at a fair representation of their evolutionary history.

\subsection{Are DSph Abundance Patterns Consistent with the Building Block Scenario?}

During the past few years more and more detailed, high-resolution abundance analyses of individual red giants in dSphs have become available, leading to a growing, yet still limited body of knowledge about their detailed element abundance ratios. In particular, $[\alpha / \mathrm{Fe}]$ ratios, $\mathrm{r}$ - and s-process abundances are being measured.

If dSphs were dominant contributors to the build-up of the Galactic halo, their abundance patterns should match those of the halo. However, the existing measurements show pronounced differences to the abundance ratios in our Galactic halo: Dwarfs are characterized by slower star formation rates, leading to reduced $[\alpha / \mathrm{Fe}]$ ratios at lower metallicity $([\mathrm{Fe} / \mathrm{H}])$ than found in the Galactic halo. This can be interpreted as a signature of a larger contribution of supernovae of Type Ia early on, such that a solar $[\alpha / \mathrm{Fe}]$ is reached sooner (e.g., Matteucci 2003). In contrast, the Galactic halo experienced comparatively rapid star formation accompanied by gas removal, leading to low metallicities with higher $\alpha$ element ratios. These different properties lead to the conclusion that dSphs cannot have contributed in a major way to the build-up of the Galactic halo (Shetrone et al. 2001), unless the majority of the minor merger events occurred at very early epochs when the abundance ratios in the Milky Way and in the dSphs were still very similar. 


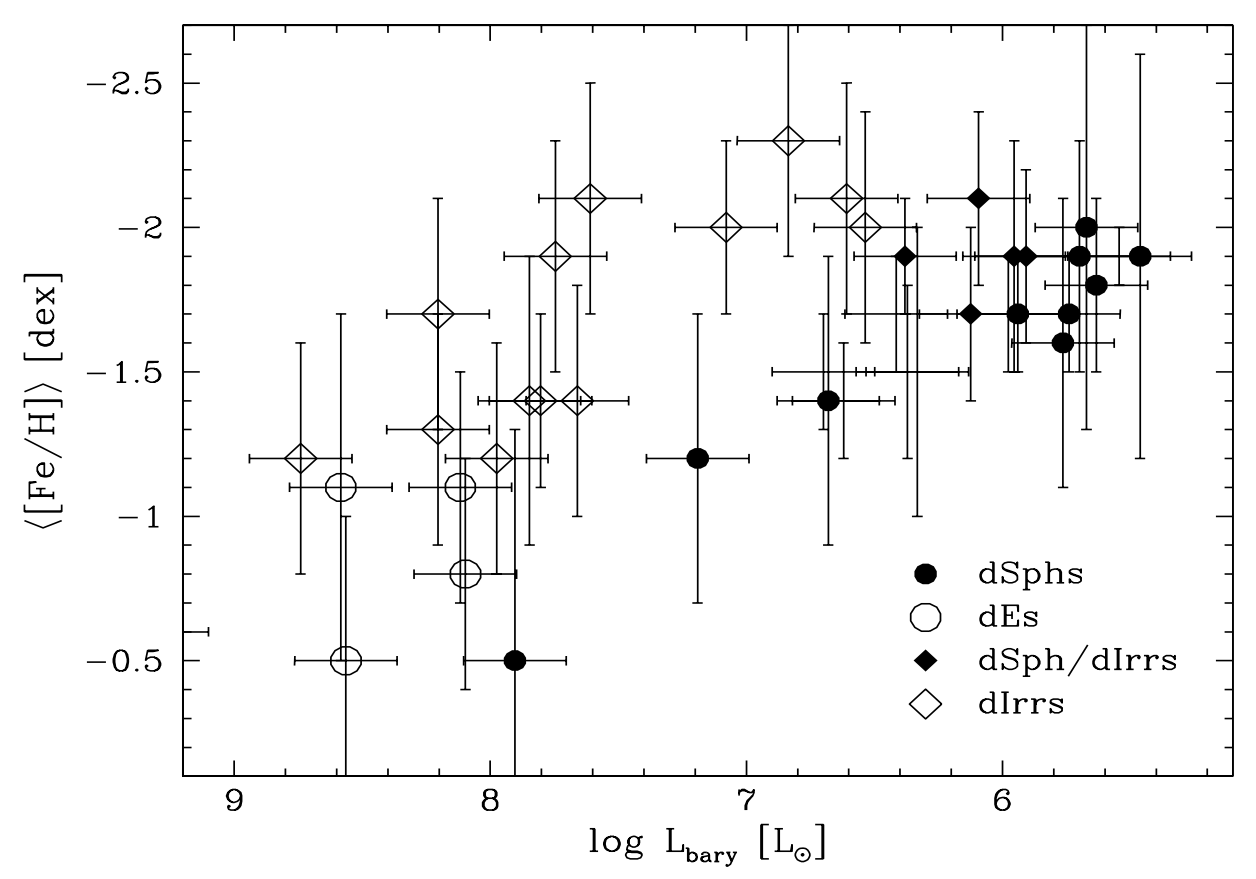

Figure 2. Mean stellar metallicity of Population II stars versus baryonic luminosity for different classes of dwarf galaxies as indicated in the legend. Note the offset between gas-deficient (filled symbols) and gas-rich (open symbols) dwarfs. At the same galaxy luminosity, the old populations of dSphs are more metal-rich than those of dIrrs. Thus in contrast to dIrrs, dSphs must have experienced comparatively rapid early enrichment. Note the location of the so-called $\mathrm{dIrr} / \mathrm{dSph}$ transition-type galaxies, which combine population properties of dSphs with ongoing star formation and a measurable gas content in the diagram. Their properties make them plausible dSph progenitors. For details, see Grebel, Gallagher, \& Harbeck (2003).

\subsection{Morphological segregation and the metallicity-luminosity relation for dwarf galaxies}

Going back to global metallicities ("[Fe/H]"), what can these tell us about galaxy evolution and interrelations between different galaxies? Now we do not consider dSphs as building blocks of larger galaxies, but dSphs as the stripped remnants of initially more massive galaxies. Clearly, dSphs must once have been more massive and considerably more gas-rich in order to have formed the stars we observe in them today. Their presentday gas deficiency still lacks a satisfactory explanation (Gallagher et al. 2003, Grebel et al. 2003). What were the progenitors of dSphs? The first type of galaxies that comes to mind are dIrrs, gas-rich, irregularly shaped dwarfs with ongoing star formation yet also with very old populations.

Could dSphs simply be stripped dIrrs? Taken at face value, the morphological segregation observed in the Local Group (as well as in other groups) would seem to support this idea (see, e.g., Fig. 1 in Grebel 1995): Gas-deficient dwarf galaxies (dEs, dSphs) are usually found within $300 \mathrm{kpc}$ around more massive galaxies, while gas-rich dwarfs (esp. dIrrs) are also (and predominantly) found at larger distances. When plotting distance from the nearest primary vs. H I content, there is a clear tendency to find dSphs with H I mass limits below $10^{5} \mathrm{M}_{\odot}$ within $300 \mathrm{kpc}$, while dIrr galaxies with typical H I masses $>10^{7} \mathrm{M}_{\odot}$ tend to lie at distances $>400 \mathrm{kpc}$ (Grebel et al. 2003, their Fig. 3). The proximity to massive galaxies and interactions with these may be an efficient agent in removing material from the dwarfs (e.g., Mayer et al. 2001). 
On the other hand, the luminosity-metallicity relations of dSphs and dIrrs have long been known to differ. While for both classes of galaxies the metallicity increases with luminosity (and hence with mass), the two relations are offset from one another (e.g., Skillman \& Bender 1995) in the sense that dSphs are more metal-rich for a given luminosity. However, the luminosity-metallicity relations are based on different tracers: For dIrrs, usually the present-day oxygen abundances as measured in $\mathrm{H}$ II regions are used, while for dEs and dSphs, metallicities of old populations (and occasionally oxygen abundances of intermediate-age planetary nebulae) are used. Thus the metallicities of populations of very different ages as well as nebular abundances versus stellar abundances are compared.

This mixture of different evolutionary stages and different metallicity indicators is unsatisfactory. Therefore we decided to attempt to compare apples with apples: In order to compare not only mean stellar metallicities in dIrrs and dSphs, but also the metallicities of the same populations (i.e., of stars of similar age), we chose old Population II giants, which are found in all LG dwarf galaxies. We used (1) old red giants in dSphs and in the outskirts of dIrrs (where old populations dominate), (2) spectroscopic abundances wherever available (from our own and literature data), and (3) photometric abundances elsewhere. The resulting data set may not yet have an ideal degree of homogeneity, but is the best and most comprehensive one currently available (Grebel et al. 2003). In the coming years, undoubtedly stellar spectroscopic measurements will also become available for those dwarfs for which we only have photometric estimates at present.

Interestingly, even when confining the comparison of luminosity-metallicity relations to old populations, the differences continue to exist. Thus at the same galaxy luminosity, the old populations of dSphs are more metal-rich than those of dIrrs. This indicates that in contrast to dIrrs, dSphs must have experienced fairly rapid early enrichment (Grebel et al. 2003). This and several other factors make dIrrs unlikely progenitors of dSphs. If dSphs are stripped remnants of more massive galaxies, then the fact that they do follow a baryonic luminosity-metallicity relation indicates that they must have continued to form stars and to experience enrichment even after the main mass removal occurred. Grebel et al. (2003) present a series of arguments why dIrr/dSph transition-type galaxies appear to be fairly plausible dSph progenitors, suggesting a gentle and slow transition from one kind of low-mass galaxy to another.

\section{Harassment and accretion}

While we presented arguments against a simple cosmological building block scenario in the preceding paragraphs, there is clear evidence for ongoing harassment and accretion of dwarf galaxies. The most prominent examples of ongoing accretion are the tidal streams of the Sagittarius dSph galaxy (Ibata et al. 1994), and the giant stream of metal-rich giants around M31 (Ibata et al. 2001). Additional stellar overdensities have been detected in the Milky Way, e.g., the Monoceros feature (Newberg et al. 2002; Yanny et al. 2003), the Canis Major overdensity (Martin et al. 2004), the Triangulum-Andromeda feature (Rocha-Pinto et al. 2004) and more substructure near M31 (Zucker et al. 2004a). These may be parts of the tidal tails of disrupted dwarfs. The continuation of deep wide-field surveys and the addition of spectroscopic data for phase-space information will ultimately permit us to identify and constrain less pronounced accretion events and their number, providing an important observable for hierarchical structure formation.

Evidence for harassment is apparent in the S-shaped surface density profile of the Galactic dSph Ursa Minor (Palma et al. 2003) and in the twisted isophotes of the M31 dE companions M32 and NGC 205 (Choi et al. 2002). These and other dSphs may eventually be accreted as well. A crucial bit of information in this context is the knowledge of the orbits of dwarf companions - something the Gaia mission will help to establish. 


\section{Concluding remarks}

What is the cosmological role of dSphs? With regard to the oldest measurable ages and the earliest epoch of star formation, we find consistency with expectations from cosmological modes. There appears to be a common epoch of early star formation in the Milky Way and in its dwarf companions. In contrast to model predictions, the expected cessation of star formation after reionization is, however, not observed in dSphs. The observed population structure in the (very different) halos of M31 and of the Milky Way, makes it seem unlikely that (present-day) dSphs played a major role in the build-up of the halo of the two spirals. The large variations in the star formation histories of dSphs and the presence of younger populations than in the Galactic halo can be reconciled with the building block scenario if most of the accretion events occurred very early on.

The global metallicities of the Milky Way dSphs are well-matched to those observed in the Galactic halo, but this is not the case for the M31 dSph companions. With regard to detailed chemical element abundance ratios, it is emerging that dSphs cannot have been dominant contributors to halo build-up unless - again - the merger events would have taken place at very early times. The differences in the metallicity-luminosity relation of different types of dwarfs seem to exclude that dSphs are simply stripped dIrrs.

Both disruption and accretion of dwarf companions are still occurring today, demonstrating that dSphs must have played some role in the growth of larger galaxies. Unfortunately, the number and importance of accretion events remains unclear for either of the two large spirals in the Local Group.

In spite of admirable progress, dwarfs remain an evolutionary puzzle. They are excellent and important test cases of cosmological predictions. Regardless of their cosmological importance, however, dwarf galaxies are also interesting in their own right!

\section{Acknowledgements}

Many thanks to Helmut Jerjen and Bruno Binggeli for a wonderful conference and for their patience while this contribution was finished. I am also indebted to Jay Gallagher for a critical reading of this text.

\section{References}

Barkana, R., \& Loeb, A. 1999, Phys. Rep. 349, 125

Bonifacio, P., Sbordone, L., Marconi, G., Pasquini, L., \& Hill, V. 2004, A\& A 414, 503

Brown, T.M., Ferguson, H.C., Smith, E., Kimble, R.A., Sweigart, A.V., Renzini, A., Rich, R.M., \& VandenBerg, D.A. 2003, ApJ 592, L17

Brown, T.M., Ferguson, H.C., Smith, E., Kimble, R.A., Sweigart, A.V., Renzini, A., \& Rich, R.M. 2004, $A J$ 127, 2738

Choi, P.I., Guhathakurta, P., \& Johnston, K.V. 2002, AJ 124, 310

Cole, A.A., Tolstoy, E., Gallagher, J.S., \& Smecker-Hane, T.A. 2005, AJ 129, 1465

Gallagher, J.S., \& Wyse, R.F.G. 1994, PASP 106, 1225

Gallagher, J.S., Madsen, G.J., Reynolds, R.J., Grebel, E.K., \& Smecker-Hane, T.A. 2003, ApJ 588,326

Grebel, E.K. 1997, Reviews in Modern Astronomy 10, 27

Grebel, E.K. 1999, in: P. Whitelock \& R. Cannon (eds.), The Stellar Content of the Local Group, IAU Symp. 192, (San Francisco: ASP), p. 17

Grebel, E.K. 2000, in: F. Favata, A.A. Kaas, \& A. Wilson (eds.), Star formation from the small to the large scale, 33rd ESLAB Symposium, ESA-SP 445 (Noordwijk: ESA), p. 87

Grebel, E.K. 2001, A\&SSSS 277, 231

Grebel, E.K. 2005, in: J. Mikolaewska \& A. Olech (eds.), Stellar Astrophysics with the World's Largest Telescopes, AIP Conf. Proc. Vol. 752 (New York: AIP), p. 161

Grebel, E.K., Gallagher, J.S., \& Harbeck, D. 2003, AJ 125, 1926 
Grebel, E.K., \& Gallagher, J.S. 2004, ApJ, 610 L89

Grebel, E.K., \& Stetson, P.B. 1999, in: P. Whitelock \& R. Cannon (eds.), The Stellar Content of the Local Group, IAU Symp. 192, (San Francisco: ASP), p. 165

Harbeck, D. et al. 2001, AJ 122, 3092

Harbeck, D., Gallagher, J.S., \& Grebel, E.K. 2004, AJ 127, 2711

Harbeck, D., Gallagher, J.S., Grebel, E.K., Koch, A., \& Zucker, D.B. 2005, ApJ 623, 159

Ikuta, C., \& Arimoto, N. 2002, A\& A 391, 55

Ibata, R.A., Gilmore, G., \& Irwin, M.J. 1994, Nature 370, 194

Ibata, R., Irwin, M., Lewis, G., Ferguson, A.M.N., \& Tanvir, N. 2001, Nature 412, 49

Karachentsev, I.D., et al. 2002, A\&A 389, 812

Klessen, R.S., Grebel, E.K., \& Harbeck, D. 2003, ApJ 589, 798

Martin, N.F., Ibata, R.A., Bellazzini, M., Irwin, M.J., Lewis, G.F., \& Dehnen, W. 2004, MNRAS 348,12

Mateo, M. 1998, ARA $\mathscr{E} A$ 36, 435

Matteucci, F. 2003, ApESSS 284, 539

Mayer, L., Governato, F., Colpi, M., Moore, B., Quinn, T., Wadsley, J., Stadel, J., \& Lake, G. 2001, ApJ 547, L123

Monelli, M., et al. 2003, $A J$ 126, 218

Newberg, H.J., et al. 2002, ApJ 569, 245

Odenkirchen, M., et al. 2001, $A J$ 122, 2538

Palma, C., Majewski, S.R., Siegel, M.H., Patterson, R.J., Ostheimer, J.C., \& Link, R. 2003, AJ 125,1352

Pont, F., Zinn, R., Gallart, C., Hardy, E., \& Winnick, R. 2004, AJ 127, 840

Rocha-Pinto, H.J., Majewski, S.R., Skrutskie, M.F., Crane, J.D., \& Patterson, R.J. 2004, ApJ 615,732

Shetrone, M.D., Côté, P., \& Sargent, W.L.W. ApJ 2001, 548, 592

Shetrone, M., Venn, K.A., Tolstoy, E., Primas, F., Hill, V., \& Kaufer, A. 2003, AJ 125, 684

Skillman, E.D., \& Bender, R. 1995, RMxAC 3, 25

Smecker-Hane, T.A., Stetson, P.B., Hesser, J.E., \& Lehnert, M.D. 1994, AJ 108, 507

Somerville, R.S. 2002, ApJ 572, L23

Stetson, P.B., Hesser, J.E., \& Smecker-Hane, T.A. 1998, PASP 110, 533

Susa, H., \& Umemura, M. 2004, ApJ 600, 1

Tolstoy, E., Irwin, M.J., Cole, A.A., Pasquini, L., Gilmozzi, R., \& Gallagher, J.S. 2001, MNRAS 327,918

Unavane, M., Wyse, R.F.G., \& Gilmore, G. 1996, MNRAS 278, 727

van den Bergh, S. 1999, A\&ARv 9, 273

van den Bergh, S. 2000, The Galaxies of the Local Group, Cambridge Astrophysics Series Vol. 35 (Cambridge: Cambridge University Press)

Wilkinson, M.I., Kleyna, J.T., Evans, N.W., Gilmore, G.F., Irwin, M.J., \& Grebel, E.K. 2004, ApJ 611, L21

Yanny, B., et al. 2003, ApJ 588, 824

Zucker, D.B., et al. 2004a, ApJ 612, L117

Zucker, D.B., et al. 2004b, ApJ 612, L121

\section{Discussion}

Conselice: What fraction of the stellar mass in Local Group dSphs formed before reionization?

GREBEL: In order to answer that question we need to determine absolute ages as opposed to relative ages, which forces us to rely on isochrone models whose oldest ages are inconsistent with the age of the Universe as inferred from WMAP results. Furthermore, we would need the ability to carry out highly accurate age determinations for individual stars in the old Population II to be able to establish when exactly they were formed before, during, or after reionization. We lack such accurate age indicators. 
FERGUSON: A pretty clear prediction is that the pre-reionization stellar population ought to have high $[\alpha / \mathrm{Fe}]$. Do the lowest metallicity stars in dSphs have high $[\alpha / \mathrm{Fe}]$ ? How well do we know high $[\alpha / \mathrm{Fe}]$ for the lowest-metallicity stars in $\mathrm{dSph}$ galaxies?

GreBeL: Metal-poor giants in dSphs have high $[\alpha / \mathrm{Fe}]$, but their values lie on average below those of Galactic stars at the same $[\mathrm{Fe} / \mathrm{H}]$ (see, e.g., the compilation in Venn et al. 2004). Very few candidates for very metal-poor giants in dSphs have been identified so far, and even fewer have had detailed high-resolution spectroscopic abundance determinations. Those for which metallicities exist tend to like below the Galactic $[\alpha / \mathrm{Fe}]$. Clearly, more data are needed.

TAYLOR: Are there well-established age-metallicity relations within individual dwarfs?

Grebel: This depends on your definition of "well-established". Synthetic color-magnitude diagram techniques provide age-metallicity relations, but purely photometric data may be affected by age-metallicity degeneracies. If instead one has the means to determine one of the ingredients through an independent method, this knowledge can be used as a fixed parameter when fitting the photometric data points. More specifically, when chemical abundances can be determined independently via spectroscopy, this knowledge can be taken into account when fitting synthetic color-magnitude diagrams to photometric data. Such studies are currently underway by several groups. Depending on the number of stars measured photometrically and spectroscopically and on the quality of the models used, well-established age-metallicity relations should result.

TAYLOR: Are there any dwarfs whose old populations look like/are consistent with the halo?

Grebel: In terms of age, yes. As I pointed out, the old Population II stars in dwarfs and in the Galactic halo are coeval within the measurement accuracy. The mean metallicities are consistent as well. With regard to $[\alpha / \mathrm{Fe}]$, there are the mentioned discrepancies. Note that this does not exclude dSph accretion; this merely means that they were not the dominant contributors to the halo build-up.

GALlART: I don't think we have enough information to exclude substantial amounts of intermediate-age populations in the Andromeda dSphs. We do not have color-magnitude diagrams for them that reach the oldest main-sequence turnoffs. My impression is that you would find at least similar amounts of intermediate-age populations in them as in M31's halo.

GREBEL: I agree that very deep color-magnitude diagrams that reach below the oldest main-sequence turnoffs are highly desirable for the M31 dSph companions. The presently available HST data only reach the horizontal branches. Hence attempts to carry out modeling with synthetic color-magnitude diagrams will be affected by substantial uncertainties. My statement that the M31 dSphs are predominantly old is, however, not based on such modeling. Instead, I am using the following indicators: (1) the absence of a red clump. (2) The absence of a clearly recognizable population of luminous AGB stars. (3) The lack of carbon stars. (4) The lack of other indicators such as a blue main sequence and a blue plume. From this I conclude that intermediate-age populations, if present at all, cannot be very numerous. 